\title{
A CLASS OF GENERALIZED MID-POINT ALGORITHMS FOR THE GURSON-TVERGAARD MATERIAL MODEL
}

\author{
Z. L. ZHANG* AND E. NIEMI
}

Department of Mechanical Engineering, Lappeenranta University of Technology, P.O. Box 20,53851 Lappeenranta, Finland

\begin{abstract}
SUMMARY
We investigate the generalized mid-point algorithms for the integration of elastoplastic constitutive equations for the pressure-dependent Gurson-Tvergaard yield model. By exact linearization of the algorithms and decomposition of the stresses into hydrostatic and deviatoric parts, a formula for explicitly calculating the consistent tangent moduli with the generalized mid-point algorithms is derived for the GursonTvergaard model. The generalized mid-point algorithms, together with the consistent tangent moduli, have been implemented into ABAQUS via the user material subroutine. An analytical solution of the GursonTvergaard model for the plane strain tension case is given and the performances of the generalized mid-point algorithms have been assessed for plane strain tension and hydrostatic tension problems and compared with the exact solutions. We find that, in the two problems considered, the generalized mid-point algorithms give reasonably good accuracy even for the case using very large time increment steps, with the true mid-point algorithm $(\alpha=0.5)$ the most accurate one. Considering the extra non-symmetrical property of the consistent tangent moduli of the algorithms with $\alpha<1$, the Euler backward algorithm $(\alpha=1)$ is, perhaps, the best choice.
\end{abstract}

KEY WORDS: Gurson model; numerical algorithms; consistent tangent moduli

\section{INTRODUCTION}

The integration of constitutive equations is the most important part of any numerical scheme employed for the analysis of elastoplastic problems. Efficient schemes which are both fast and accurate are needed. The algorithms employed for the integration of constitutive equations can be classified into two groups: those based on an explicit technique and those based on an implicit technique. Recently, implicit algorithms, falling within the category of return mapping algorithms, have become more and more popular. ${ }^{1-5}$ Within the framework of operator splitting methodology, Simo and Ortiz $^{6}$ have proposed a new class of return mapping algorithms applicable to a general class of plastic and viscoplastic constitutive models.

In recent years, there has been growing interest in the analysis of plastic flow localization and fracture behaviour of ductile porous metals. Unlike the conventional von Mises model, however, the yield models for porous solids exhibit a dependence on hydrostatic pressure. It is now well established that the fracture of ductile metals results from the initiation, growth and coalescence of microscopic voids. In order to accurately predict the limit to ductility of structural metals, it is necessary to have a constitutive theory which properly incorporates the inelastic straining resulting from the nucleation and growth of voids. Gurson ${ }^{7,8}$ has developed a theory of dilatational plasticity for this purpose, which has been modified by Tvergaard ${ }^{9,10}$ in order to

" Current address: SINTEF Materials Technology, N-7034 Trondheim, Norway.

CCC 0029-5981/95/122033-21

Received 9 August 1993

(C) 1995 by John Wiley \& Sons, Ltd.

Revised 30 August 1994 
bring it into better correspondence with his numerical studies. The Gurson-Tvergaard modified model has been applied more than any other model for the studies of the failure of ductile metals. ${ }^{11}$ In the Gurson-Tvergaard model, not only the hydrostatic pressure but the material damage parameter, void volume fraction $f$, has been incorporated into the yield function. It is known that an essential ingredient in the overall performance of the numerical integration algorithm is the development of consistent tangent moduli, obtained by linearization of the numerical integration algorithm. Due to the non-trivial task of obtaining the consistent tangent moduli with return mapping algorithms for the Gurson-Tvergaard model, Euler forward algorithms, based on the use of elastoplastic tangent moduli, are widely used.9,10,12-14 However, recently Worswick and Pick ${ }^{15}$ have indicated that the application of the Euler forward algorithm for the Gurson-Tvergaard model requires very small time steps in order to avoid numerical instability. A Euler backward integration algorithm for the Gurson-Tvergaard model has been presented by Aravas. ${ }^{16}$ Aravas also gave a formulation for the calculation of the consistent tangent moduli with his Euler backward algorithm. However, the calculation of the consistent tangent moduli is very complicated and sometimes impossible. Recently an explicit expression for the consistent tangent moduli with the Euler backward algorithm for the Gurson-Tvergaard model has been given by Zhang. ${ }^{17}$ Zhang $^{17,18}$ has also studied the accuracies of the return mapping algorithms using iso-error maps, by formulating a class of generalized mid-point algorithms for the Gurson-Tvergaard model.

In this paper, the generalized mid-point algorithms formulated in Reference 18 are modified in order to derive the consistent tangent moduli. Within the framework of the return mapping algorithms, and based on the decomposition of stress increments into hydrostatic and deviatoric components, a seven-constant formula for explicitly calculating the tangent moduli consistent with the generalized mid-point algorithms is presented for the Gurson-Tvergaard model. Like the methodology in the Euler backward algorithm, ${ }^{17}$ the significant advantage of this methodology is that no matrix inversion is required in the consistent tangent moduli expression. These generalized mid-point algorithms have been incorporated into the finite element program ABAQUS via the user material subroutine. The performances of the generalized mid-point algorithms have been assessed for practical plane strain tension and hydrostatic tension problems, and compared with exact solutions. General conclusions about the choice of the algorithms are given.

\section{GURSON-TVERGAARD MATERIAL MODEL AND TWO SOLUTIONS}

A detailed review of the continuum mechanics framework for analysing plastic flow localization has been recently given by Needleman and Tvergaard. ${ }^{19}$ Under quasi-static loading conditions, it is believed that the material constitutive features play an important role in the initiation of shear bands. It has been found that the von Mises model with a smooth yield surface is quite resistant to localization and, as a consequence, localization predictions are very sensitive to the deviations from this classical constitutive description. It has been shown that the Gurson-Tvergaard model, which can incorporate the explicit softening processes, such as the softening arising from microvoid nucleation and growth, has been getting increasingly popular for simulating plastic flow localization and ductile fracture. The Gurson-Tvergaard constitutive model is the object of this study. Obviously the method presented in this paper can be easily extended to other material models, for example, to the lower bound yield model. ${ }^{20,21}$ In the following text, a brief summary of the Gurson-Tvergaard model for completeness is provided first. Then two case solutions of the Gurson-Tvergaard model are given. 


\subsection{Gurson-Tvergaard material model}

Based on an approximation of a solid with a volume fraction $f$, of voids, as a spherical body with a concentric spherical void, and carrying out an approximate rigid-plastic upper bound analysis of this thick-walled spherical cell, with matrix material idealized as rigid-perfectly plastic and obeying the von Mises yield criterion, Gurson ${ }^{7,8}$ obtained the following dilational yield condition $\left(q_{1}=1\right.$ and $\left.q_{2}=1\right)$ for a porous plastic solid, which is a good fit to his upper bound yield load locus:

$$
\phi\left(\sigma, f, \sigma_{f}\right)=\frac{q^{2}}{\sigma_{f}^{2}}+2 q_{1} f \cosh \left(-\frac{3 q_{2} p}{2 \sigma_{f}}\right)-\left(1+q_{1}^{2} f^{2}\right)=0
$$

where constants $q_{1}$ and $q_{2}$ were introduced by Tvergaard ${ }^{9,10}$ to bring predictions of the model into closer agreement with his full numerical analyses of a periodic array of voids. $\sigma_{f}$ is the average matrix flow stress; $q$ and $p$ are the effective and pressure parts of the average macroscopic Cauchy stress $\sigma$. It is easy to see that the material loses load carrying capacity if $f$ reaches the limit $1 / q_{1}$, because all the stress components have to vanish in order to satisfy (1). It is clear that the effect of Tvergaard's modification is merely to decrease the critical void volume fraction $f_{c}$, at which the material will lose load carrying capacity. Since only for small values of $f$, the equivalent von Mises stress becomes close to the flow stress, it is evident that the Gurson-Tvergaard model is strongly dependent on the ratio of the hydrostatic stress to the flow stress.

In general the evolution of the microvoid volume fraction results from the growth of existing microvoids and the nucleation of new microvoids can be written as

$$
\mathrm{d} f=\mathrm{d} f_{\text {growth }}+\mathrm{d} f_{\text {nucleation }}
$$

The increase of the void volume fraction due to the growth of existing voids is determined from the condition that the matrix material is plastically incompressible such that

$$
\mathrm{d} f_{\text {growth }}=(1-f) \mathrm{d} \varepsilon^{\mathrm{p}}: \mathbf{I}
$$

The contribution resulting from the nucleation of new voids is usually taken to be either strain controlled or stress controlled. Here, we only consider the plastic strain controlled case as

$$
\mathrm{d} f_{\text {nucleation }}=A \mathrm{~d} \bar{\varepsilon}^{\mathrm{p}}
$$

where $\bar{\varepsilon}^{\mathrm{p}}$ is the equivalent plastic strain in the matrix material. The parameter $A$ is chosen such that void nucleation follows a normal distribution as suggested by Chu and Needleman: ${ }^{22}$

$$
A=\frac{f_{\mathrm{N}}}{S_{\mathrm{N}} \sqrt{2 \pi}} \exp \left[-\frac{1}{2}\left(\frac{\bar{\varepsilon}^{\mathrm{p}}-\varepsilon_{\mathrm{N}}}{S_{\mathrm{N}}}\right)^{2}\right]
$$

with $f_{\mathrm{N}}$ the volume fraction of void-nucleating particles, $\varepsilon_{\mathrm{N}}$ the mean strain for void nucleation and $S_{\mathrm{N}}$ the corresponding standard deviation. Moreover, the equivalence of the overall rate of plastic work and that in the matrix material leads to

$$
\boldsymbol{\sigma}: \mathrm{d} \varepsilon^{\mathfrak{p}}=(1-f) \sigma_{f} \mathrm{~d} \bar{\varepsilon}^{\mathrm{p}}
$$

or equivalently

$$
\mathrm{d} \bar{\varepsilon}^{\mathrm{p}}=\frac{\sigma: \mathrm{d} \varepsilon^{\mathrm{p}}}{(1-f) \sigma_{f}}
$$


The plastic part of the macroscopic strain increment is taken to be proportional to the normal $\partial \phi / \partial \boldsymbol{\sigma}$ of the yield function:

$$
\mathrm{d} \varepsilon^{\mathrm{p}}=\mathrm{d} \lambda \frac{\partial \phi}{\partial \boldsymbol{\sigma}}
$$

\subsection{Solution for plane strain tension problem}

We shall present the solution of the Gurson-Tvergaard model to the plane strain tension problem. Figure 1 shows an element under plane strain tension with a Cartesian co-ordinate system $(x, y, z)$ defined in such a way that $y$ is the tensile axis. In Figure $1 a_{0}$ is the initial length of the element. The only unknown stress for the problem is $\sigma_{y}$, as $\sigma_{z}$ is a function of $\sigma_{y}$. Using the equations just described and enforcing the consistency condition to determine $\mathrm{d} \lambda$ we obtain, after some algebraic manipulations, the following equations describing the problem:

$$
\begin{aligned}
\frac{\mathrm{d} \varepsilon_{y}^{\mathrm{p}}}{\mathrm{d} \varepsilon_{y}} & =\omega \phi_{\sigma_{y}}, \quad \frac{\mathrm{d} \varepsilon_{z}^{\mathrm{p}}}{\mathrm{d} \varepsilon_{y}}=\omega \phi_{\sigma_{z}} \\
\frac{\mathrm{d} \sigma_{y}}{\mathrm{~d} \varepsilon_{y}} & =\frac{E}{1-v^{2}}\left[1-\frac{\mathrm{d} \varepsilon_{y}^{\mathrm{p}}}{\mathrm{d} \varepsilon_{y}}-v \frac{\mathrm{d} \varepsilon_{y}^{\mathrm{p}}}{\mathrm{d} \varepsilon_{y}}\right], \quad \frac{\mathrm{d} \sigma_{z}}{\mathrm{~d} \varepsilon_{y}}=v \frac{\mathrm{d} \sigma_{y}}{\mathrm{~d} \varepsilon_{y}}-E \frac{\mathrm{d} \varepsilon_{y}^{\mathrm{p}}}{\mathrm{d} \varepsilon_{y}} \\
\frac{\mathrm{d} f}{\mathrm{~d} \varepsilon_{y}} & =\Lambda_{f} \omega \\
\frac{\mathrm{d} \bar{\varepsilon}^{\mathrm{p}}}{\mathrm{d} \varepsilon_{y}} & =\Lambda_{\bar{\varepsilon}} \omega
\end{aligned}
$$

where

$$
\begin{aligned}
\omega & =\frac{E\left(\phi_{\sigma_{y}}+\phi_{\sigma_{z}}\right)}{1-v^{2}} /\left[\frac{E\left(\phi_{\sigma_{y}}^{2}+\phi_{\sigma_{z}}^{2}+2 v \phi_{\sigma_{y}} \phi_{\sigma_{z}}\right)}{1-v^{2}}-\phi_{f} \Lambda_{f}-\phi_{\sigma_{f}} \frac{\mathrm{d} \sigma_{f}}{\mathrm{~d} \bar{\varepsilon}^{\mathrm{p}}} \Lambda_{\bar{\varepsilon}}\right] \\
\phi_{\sigma_{y}} & =\frac{q_{1} q_{2} f}{\sigma_{f}} \sinh \left(-\frac{3 q_{2} p}{2 \sigma_{f}}\right)+\frac{2 \sigma_{y}-\sigma_{z}}{\sigma_{f}^{2}}
\end{aligned}
$$

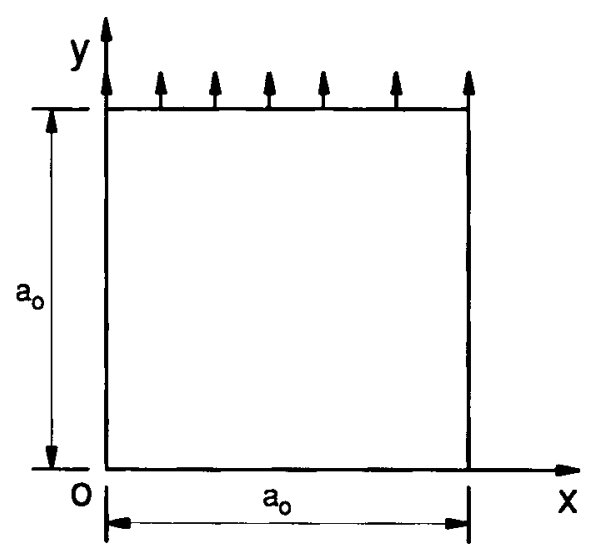

Figure 1. Plane strain tension 


$$
\begin{aligned}
\phi_{\sigma_{z}} & =\frac{q_{1} q_{2} f}{\sigma_{f}} \sinh \left(-\frac{3 q_{2} p}{2 \sigma_{f}}\right)+\frac{2 \sigma_{z}-\sigma_{y}}{\sigma_{f}^{2}} \\
\phi_{f} & =2 q_{1} \cosh \left(-\frac{3 q_{2} p}{2 \sigma_{f}}\right)-2 q_{1}^{2} f \\
\phi_{\sigma_{f}} & =-\frac{2 q^{2}}{\sigma_{f}^{3}}+\frac{3 q_{1} q_{2} f p}{\sigma_{f}^{2}} \sinh \left(-\frac{3 q_{2} p}{2 \sigma_{f}}\right) \\
\Lambda_{\bar{\varepsilon}} & =\frac{\left(\phi_{\sigma_{y}} \sigma_{y}+\phi_{\sigma_{z}} \sigma_{z}\right)}{(1-f) \sigma_{f}} \\
\Lambda_{f} & =(1-f) \frac{3 q_{1} q_{2} f}{\sigma_{f}} \sinh \left(-\frac{3 q_{2} p}{2 \sigma_{f}}\right)+A \Lambda_{\bar{\varepsilon}} \\
q & =\sqrt{\sigma_{y}^{2}+\sigma_{z}^{2}-\sigma_{y} \sigma_{z}} \\
p & =-\frac{\sigma_{y}+\sigma_{z}}{3}
\end{aligned}
$$

$E$ and $v$ are Young's modulus and Poisson's ratio, respectively, $\varepsilon_{y}$ and $\varepsilon_{y}^{\mathrm{p}}$ are the strain and plastic strain in the $y$-direction and $\varepsilon_{z}^{p}$ is the plastic strain in the $z$-direction.

\subsection{Solution for hydrostatic tension problem}

This problem has been solved by Aravas. ${ }^{16}$ Figure 2 shows the element dimensions. The derivation of the equations is simpler than the plane strain case. We can easily get the following equations describing the problem, which can be integrated numerically:

$$
\frac{\mathrm{d} \sigma_{x}}{\mathrm{~d} \varepsilon_{\mathrm{v}}}=\frac{\mathrm{K} \Lambda}{\Lambda+9 \beta^{2} K}
$$

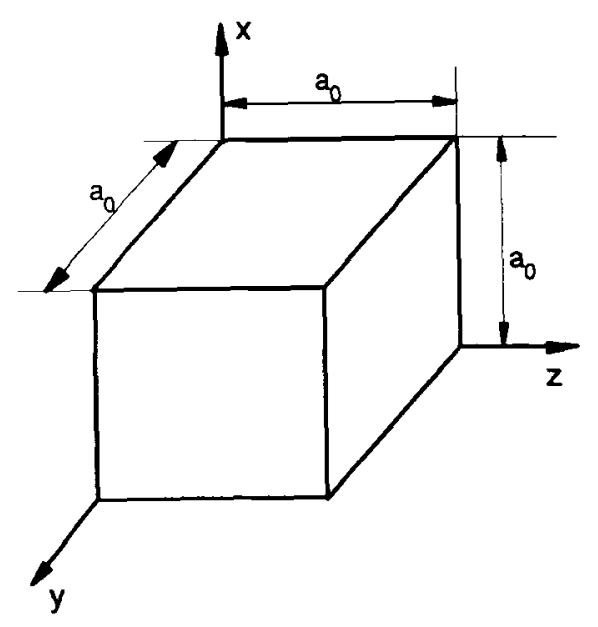

Figure 2. Hydrostatic tension 


$$
\begin{aligned}
\frac{\mathrm{d} \bar{\varepsilon}^{\mathrm{p}}}{\mathrm{d} \varepsilon_{\mathrm{v}}} & =\frac{3 \beta^{2} K}{\Lambda+9 \beta^{2} K} \frac{\varpi}{1-f} \\
\frac{\mathrm{d} f}{\mathrm{~d} \varepsilon_{\mathrm{v}}} & =\left[3(1-f)+\frac{A \varpi}{1-f}\right] \frac{3 \beta^{2} K}{\Lambda+9 \beta^{2} K}
\end{aligned}
$$

where $K$ is the bulk modulus, $\varepsilon_{v}$ is the logarithmic volumetric strain, $\varepsilon_{v}=3 \ln \left(1+u / a_{0}\right), u$ is the prescribed displacement and

$$
\begin{aligned}
& \boldsymbol{\varpi}=\frac{3 \sigma_{x}}{\sigma_{f}} \\
& \Lambda=\frac{\beta \varpi}{1-f}\left[\frac{\mathrm{d} \sigma_{f}}{\mathrm{~d} \bar{\varepsilon}^{\mathrm{p}}} \beta \varpi-A \frac{\phi_{f}}{2} \sigma_{f}\right]-3 \sigma_{f} \beta \frac{\phi_{f}}{2}(1-f) \\
& \beta=\frac{1}{2} q_{1} q_{2} f \sinh \left(\frac{1}{2} q_{2} \varpi\right)
\end{aligned}
$$

A small error in Reference 16 has been corrected in the expression of $\Lambda$.

\section{GENERALIZATION OF ELASTOPLASTIC CONSTITUTIVE RELATIONS}

The same elastoplastic constitutive relations as in Reference 18 are used here. For completeness and simplicity, this section just presents the related formulas. The class of elastoplastic materials considered here can be characterized by means of the following equations:

$$
\begin{aligned}
\boldsymbol{\varepsilon} & =\boldsymbol{\varepsilon}^{\mathrm{e}}+\boldsymbol{\varepsilon}^{\mathrm{p}} \\
\mathrm{d} \varepsilon & =\mathrm{d} \boldsymbol{\varepsilon}^{\mathrm{e}}+\mathrm{d} \varepsilon^{\mathrm{p}} \\
\boldsymbol{\sigma} & =\mathbf{D}^{\mathrm{e}}: \varepsilon^{\mathrm{e}} \\
\phi(\boldsymbol{\sigma}, \mathbf{H}) & =\phi(p, q, \mathbf{H})=0 \\
\mathrm{~d} \boldsymbol{\varepsilon}^{\mathfrak{p}} & =\mathrm{d} \lambda \frac{\partial g}{\partial \boldsymbol{\sigma}} \\
\mathrm{d} \mathbf{H} & =\mathbf{h}\left(\mathrm{d} \boldsymbol{\varepsilon}^{\mathrm{p}}, \boldsymbol{\sigma}, \mathbf{H}\right)=\mathbf{h}\left(\mathrm{d} \varepsilon^{\mathrm{p}}, p, q, \mathbf{H}\right)
\end{aligned}
$$

where, following standard notation, $\varepsilon, \varepsilon^{\mathbf{e}}$ and $\varepsilon^{\mathfrak{p}}$ denote the total, elastic and plastic strain tensors, respectively, and $\mathbf{H}$ signifies some internal state variables. In the paper, linear isotropic elasticity is assumed:

$$
D_{i j k l}^{\mathrm{e}}=\left(K-\frac{2}{3} G\right) \delta_{i j} \delta_{k l}+2 G \delta_{i k} \delta_{j l}
$$

where $\delta$ 's are the Kronecker delta and $G$ is the elastic shear modulus. In the general yield function (16d), $p$ and $q$ can be calculated:

$$
\begin{aligned}
& p=-\frac{1}{3} \boldsymbol{\sigma}: \mathbf{I} \\
& q=\sqrt{\frac{3}{2} \mathbf{S}: \mathbf{S}}
\end{aligned}
$$

$\mathbf{I}$ and $\mathbf{S}$ are the second-order unit tensor and deviatoric stress, respectively

The plastic flow rule is given by (16e), where $\mathrm{d} \lambda$ is a positive scalar and $g$ is the flow potential. In the literature, the associated flow rule is commonly applied to porous solids. Here, the associated 
flow rule alone is used, which means $g=\phi$. However, there are no difficulties in applying the method to the non-associated flow rule. Equation (16f) expresses the general internal state variable evolution rule, which could include both plasticity and damage evolution rules. For rate-independent materials, $\mathbf{h}$ must be homogeneous of degree one in $\mathrm{d} \varepsilon^{\mathbf{p}}$.

\section{A CLASS OF GENERALIZED MID-POINT ALGORITHMS}

A class of generalized mid-point algorithms has been formulated by Zhang $^{18}$ for the pressure-dependent Gurson-Tvergaard model. It was found later that it is difficult to derive the corresponding consistent tangent moduli for the generalized mid-point algorithms, because of the simplification made in Reference 18 . This difficulty has been overcome in this paper by a modification such that it is possible to derive the consistent tangent moduli. Here and subsequently, the subindice $n+\alpha$ is used to identify the value of the various state variables at time $t_{n+a}$.

The generalized mid-point algorithms, in the context of displacement finite element analysis, take the following forms: ${ }^{2}$

$$
\begin{aligned}
\boldsymbol{\varepsilon}_{n+1} & =\varepsilon_{n}+\Delta \varepsilon_{n+1} \\
\sigma_{n+1}^{\mathrm{T}} & =\mathbf{D}^{\mathrm{e}}:\left(\varepsilon_{n}^{\mathrm{e}}+\Delta \varepsilon_{n+1}\right) \\
\boldsymbol{\sigma}_{n+1} & =\boldsymbol{\sigma}_{n+1}^{\mathrm{T}}-\mathbf{D}^{\mathrm{e}}: \Delta \boldsymbol{\varepsilon}_{n+1}^{\mathrm{p}} \\
\Delta \boldsymbol{\varepsilon}_{n+1}^{\mathrm{p}} & =\Delta \lambda \mathbf{r}_{n+\alpha} \\
\mathbf{H}_{n+1} & =\mathbf{H}_{n}+\Delta \mathbf{H}_{n+1} \\
\Delta \mathbf{H}_{n+1} & =\mathbf{h}_{n+\alpha} \\
\phi_{n+1} & =0
\end{aligned}
$$

where one writes

$$
\begin{aligned}
\mathbf{r}_{n+\alpha} & =\left(\frac{\partial \phi}{\partial \sigma}\right)_{n+\alpha}=\mathbf{r}\left(\sigma_{n+\alpha}, \mathbf{H}_{n+\alpha}\right) \\
\mathbf{h}_{n+\alpha} & =\mathbf{h}\left(\boldsymbol{\sigma}_{n+\alpha}, \mathbf{H}_{n+\alpha}\right) \\
\boldsymbol{\sigma}_{n+\alpha} & =(1-\alpha) \boldsymbol{\sigma}_{n}+\alpha \boldsymbol{\sigma}_{n+1} \\
\mathbf{H}_{n+\alpha} & =(1-\alpha) \mathbf{H}_{n}+\alpha \mathbf{H}_{n+1}
\end{aligned}
$$

and the algorithmic parameter $\alpha$ takes values in the interval $[0,1]$. For $\alpha=1$, one obtains the closest point return algorithm or the Euler backward algorithm, ${ }^{16}$ whereby the elastic predictor is relaxed into the closest point on the updated surface. A theoretical analysis given by Ortiz and Popov $^{2}$ shows that the algorithm is second-order accurate for $\alpha=0.5$ and unconditionally stable for $\alpha \geqslant 0.5$ regardless of the shape of the yield surface.

For the constitutive model considered, we can separate any stress tensor into its deviatoric and hydrostatic components

$$
\boldsymbol{\sigma}_{n+1}=-p_{n+1} \mathbf{I}+\mathbf{S}_{n+1}=-p_{n+1} \mathbf{I}+\frac{2}{3} q \mathbf{n}_{n+1}
$$

where

$$
\mathbf{n}_{n+1}=\frac{3}{2 q} \mathbf{S}_{n+1}
$$


is the unit vector in the deviatoric space normal to the yield surface and $\mathbf{S}$ is the deviatoric stress. Likewise, the plastic strain increment can be decoupled into volumetric $\left(\Delta \varepsilon_{V}^{p}\right)$ and deviatoric $\left(\Delta \varepsilon_{\mathrm{D}}^{\mathrm{p}}\right)$ parts:

$$
\Delta \varepsilon^{\mathrm{p}}=\Delta \varepsilon_{\mathrm{V}}^{\mathrm{p}}+\Delta \varepsilon_{\mathrm{D}}^{\mathrm{p}}=\Delta \lambda\left[-\left(\frac{\partial \phi}{\partial p}\right)_{n+\alpha} \frac{1}{3} \mathbf{I}+\left(\frac{\partial \phi}{\partial q}\right)_{n+\alpha} \frac{\frac{3}{2}}{\frac{\mathbf{S}_{n+\alpha}}{q_{n+\alpha}}}\right]
$$

where the subindice $n+1$ of $\Delta \varepsilon^{p}$ is omitted for convenience. The above equation with $\alpha=1$ was used by Aravas in the Euler backward algorithm formulations. ${ }^{16}$ The following equation was used by Zhang $^{18}$ in formulating the generalized mid-point algorithms:

$$
\Delta \boldsymbol{\varepsilon}^{\mathrm{p}}=\Delta \lambda\left[-\left(\frac{\partial \phi}{\partial p}\right)_{n+\alpha} \frac{1}{3} \mathbf{I}+\left(\frac{\partial \phi}{\partial q}\right)_{n+\alpha} \frac{q_{n+1}^{\mathrm{T}}}{q_{n+\alpha}} \frac{3}{2} \frac{\mathbf{S}_{n+\alpha}}{q_{n+1}^{\mathbf{T}}}\right]
$$

where $q_{n+1}^{\mathrm{T}}$ is obtained from $\sigma_{n+1}^{\mathrm{T}}$ by equation (18b). However, one still faces difficulties when applying (23) to derive the corresponding consistent explicit tangent moduli. In this paper, we found that using the following equation guarantees the derivation of the explicit consistent tangent moduli:

$$
\Delta \mathbf{\varepsilon}^{\mathbf{p}}=\Delta \lambda\left[-\left(\frac{\partial \phi}{\partial p}\right)_{n+\alpha} \frac{1}{3} \mathbf{I}+\left(\frac{\partial \phi}{\partial q}\right)_{n+\alpha} \frac{q_{n}}{q_{n+\alpha}} \frac{3}{2} \frac{\mathbf{S}_{n+\alpha}}{q_{n}}\right]=\frac{1}{3} \Delta \varepsilon_{p} \mathbf{I}+\frac{3}{2} \Delta \varepsilon_{q} \frac{\mathbf{S}_{n+\alpha}}{q_{n}}
$$

where

$$
\begin{aligned}
\Delta \varepsilon_{p} & =-\Delta \lambda\left(\frac{\partial \phi}{\partial p}\right)_{n+\alpha} \\
\Delta \varepsilon_{q} & =\Delta \lambda \frac{q_{n}}{q_{n+\alpha}}\left(\frac{\partial \phi}{\partial q}\right)_{n+\alpha}
\end{aligned}
$$

Please note the differences between (22), (23) and (24). With (22), we have no simple equation (30b) so that $\partial q_{n+1} / \partial \Delta \varepsilon_{q}$ can be directly solved. This makes the generalized mid-point algorithms impossible. Secondly, using (23) instead of (24), equation (38) would not be available and the deriving of the explicit consistent tangent moduli would be extremely difficult, if not impossible.

By eliminating $\Delta \lambda$ from the above two equations one obtains

$$
\Delta \varepsilon_{p}\left[\frac{q_{n}}{q_{n+\alpha}}\left(\frac{\partial \phi}{\partial q}\right)_{n+\alpha}\right]+\Delta \varepsilon_{q}\left(\frac{\partial \phi}{\partial p}\right)_{n+\alpha}=0
$$

or more simply

$$
\Delta \varepsilon_{p} P+\Delta \varepsilon_{q} Q=0
$$

where

$$
\begin{aligned}
& P=\frac{q_{n}}{q_{n+\alpha}}\left(\frac{\partial \phi}{\partial q}\right)_{n+\alpha} \\
& Q=\left(\frac{\partial \phi}{\partial p}\right)_{n+\alpha}
\end{aligned}
$$

Substituting (24) into (19c) yields

$$
\boldsymbol{\sigma}_{n+1}=\boldsymbol{\sigma}_{n+1}^{\mathrm{T}}-K \Delta \varepsilon_{p} \mathbf{I}-3 G \Delta \varepsilon_{q} \frac{\mathbf{S}_{n+\alpha}}{q_{n}}
$$


By separating $\sigma_{n+1}$ and $\sigma_{n+1}^{\mathrm{T}}$ into deviatoric and hydrostatic components according to (20) and using the mid-point rule for $S_{n+\alpha}$, we obtain

$$
\begin{aligned}
& p_{n+1}=p_{n+1}^{\mathrm{T}}+K \Delta \varepsilon_{p} \\
& \mathbf{S}_{n+1}=\mathbf{S}_{n+1}^{\mathrm{T}}-3 G \Delta \varepsilon_{q} \frac{\mathbf{S}_{n+\alpha}}{q_{n}}
\end{aligned}
$$

where $p_{n+1}^{\mathrm{T}}$ and $\mathbf{S}_{n+1}^{\mathrm{T}}$ are obtained from $\boldsymbol{\sigma}_{n+1}^{\mathrm{T}}$. After obtaining $\mathbf{S}_{n+1}, q_{n+1}$ can be computed through (18b).

In the present mid-point algorithms the general internal state variable evolution rules (19f) can be written more specifically:

$$
\Delta \mathbf{H}_{n+1}=\mathbf{h}_{n+\alpha}\left(\Delta \varepsilon_{p}, \Delta \varepsilon_{q}, p_{n+\alpha}, q(\mathbf{S})_{n+\alpha}, \mathbf{H}_{n+\alpha}\right)
$$

Through the above preliminary work, the integration problem of the elastoplastic equations can be reduced to the solution of the following non-linear equations:

$$
\begin{aligned}
\Delta \varepsilon_{p} P & +\Delta \varepsilon_{q} Q=0 \\
\phi_{n+1} & =\phi\left(p_{n+1}, q(\mathbf{S})_{n+1}, \mathbf{H}_{n+1}\right)=0 \\
p_{n+1} & =p_{n+1}^{\mathbf{T}}+K \Delta \varepsilon_{p} \\
\mathbf{S}_{n+1} & =\mathbf{S}_{n+1}^{\mathrm{T}}-3 G \Delta \varepsilon_{q} \mathbf{S}_{n+\alpha} / q_{n} \\
\Delta \mathbf{H}_{n+1} & =\mathbf{h}_{n+\alpha}\left(\Delta \varepsilon_{p}, \Delta \varepsilon_{q}, p_{n+\alpha}, q(\mathbf{S})_{n+\alpha}, \mathbf{H}_{n+\alpha}\right)
\end{aligned}
$$

These equations can be solved by Newton's method using $\rho_{p}$ and $\rho_{q}$ as the corrections for $\Delta \varepsilon_{p}$ and $\Delta \varepsilon_{q}$ and treating (32a) and (32b) as the two basic equations in which $p, \mathbf{S}$ and $\Delta \mathbf{H}$ are updated by (32c)-(32e), we obtain the following two scalar Newton equations:

$$
\begin{aligned}
& A_{11} \rho_{p}+A_{12} \rho_{q}=b_{1} \\
& A_{21} \rho_{p}+A_{22} \rho_{q}=b_{2}
\end{aligned}
$$

where the constants $A_{i j}$ and $b_{i}$ are given in Appendix I. Taking $\Delta \varepsilon_{p}$ and $\Delta \varepsilon_{q}$ as zero in the beginning of the solution, these equations are solved for $\rho_{p}$ and $\rho_{q}$; and the values of $\Delta \varepsilon_{p}$ and $\Delta \varepsilon_{q}$ are updated by

$$
\begin{aligned}
\Delta \varepsilon_{p} & =\Delta \varepsilon_{p}+\rho_{p} \\
\Delta \varepsilon_{q} & =\Delta \varepsilon_{q}+\rho_{q}
\end{aligned}
$$

Then, the values $p, \mathbf{S}$ and $\Delta \mathbf{H}$ are updated using (32c)-(32e). The iteration is continued until the solution converges. In the numerical problem presented here, the convergence is controlled by the requirement $\left|\phi_{n+1}\right| \leqslant 1 \mathrm{E}-7$.

\section{EXPLICIT CONSISTENT TANGENT MODULI WITH THE GENERALIZED MID-POINT ALGORITHMS}

It has been shown ${ }^{5}$ that for rate-independent elastoplastic problems, the consistency between the tangent moduli and the integration algorithms employed in the solution of the incremental problem plays a crucial role in preserving the quadratic rate of asymptotic convergence of iterative solution schemes based on Newton's method. The so-called consistent tangent moduli are defined by exact linearization of the algorithms, (19c) and enforcing the plasticity consistency 
condition at the end of the step $t_{n+1}$ :

$$
\mathrm{D}^{\text {consis. }}=\left(\frac{\partial \sigma}{\partial \mathbf{\varepsilon}}\right)_{n+1}
$$

$D^{\text {consis. }}$ depends on the algorithm used for the integration of the constitutive equations and is, in general, different from the so-called 'elastoplastic tangent moduli' derived from the 'continuum' rate equations by enforcement of the consistency condition. ${ }^{5}$ An explicit expression of the consistent tangent moduli with the Euler backward algorithm $(\alpha=1)$ has been given by Zhang. ${ }^{17}$ In the following, we shall derive the explicit expression for the consistent tangent moduli with the whole family of the generalized mid-point algorithms proposed. Based on the decomposition of the return mapping process described above, we will derive the consistent tangent moduli separately from both the hydrostatic and deviatoric components. First we consider the deviatoric stress plane in which we have

$$
\mathbf{S}_{n+1}=\mathbf{S}_{n+1}^{\mathrm{T}}-3 G \Delta \varepsilon_{q} \frac{\mathbf{S}_{n+\alpha}}{q_{n}}
$$

By differentiating the above equation and rearranging we obtain

$$
\left(1+\frac{\left(3 G \alpha \Delta \varepsilon_{q}\right.}{q_{n}}\right) \partial \mathbf{S}_{n+1}=\partial \mathbf{S}_{n+1}^{\mathbf{T}}-3 G \frac{\mathbf{S}_{n+\alpha}}{q_{n}} \partial \Delta \varepsilon_{q}
$$

Substituting $\partial \mathbf{S}_{n+1}^{\mathrm{T}}=2 G \partial \varepsilon_{\mathrm{D}}$, where $\varepsilon_{\mathrm{D}}$ is the deviatoric component of $\varepsilon_{n+1}$, into the last equation gives

$$
\partial \mathbf{S}_{n+1}=\frac{2 G}{1+3 G \alpha \Delta \varepsilon_{q} / q_{n}}\left[\partial \mathbf{\varepsilon}_{\mathrm{D}}-\frac{3}{2} \frac{\mathbf{S}_{n+\alpha}}{q_{n}} \partial \Delta \varepsilon_{q}\right]
$$

For all the cases in which three direct strains are defined by the kinematic solution we have

$$
\begin{gathered}
\partial \boldsymbol{\varepsilon}_{\mathrm{D}}=\left[\mathbf{J}-\frac{1}{3} \mathbf{I} \otimes \mathbf{I}\right]: \partial \boldsymbol{\varepsilon} \\
\partial p_{n+1}=-K \mathbf{I}: \partial \boldsymbol{\varepsilon}+K \partial \varepsilon_{p} \\
\partial \boldsymbol{\sigma}_{n+1}=-\partial p_{n+1} \mathbf{I}+\partial \mathbf{S}_{n+1}
\end{gathered}
$$

where $\mathbf{J}$ is the fourth-order unit tensor. By substituting $\partial \mathbf{S}_{n+1}$ and $\partial p_{n+1}$ into (41) and rearranging we obtain

$$
\partial \boldsymbol{\sigma}=\mathbf{Z}: \partial \boldsymbol{\varepsilon}-K \mathbf{I} \partial \Delta \varepsilon_{p}-\frac{2 G}{1+3 G \alpha \Delta \varepsilon_{q} / q_{n}} \frac{q_{n+\alpha}}{q_{n}} \mathbf{n}_{n+\alpha} \partial \Delta \varepsilon_{q}
$$

where

$$
\mathbf{Z}=\frac{2 G}{1+3 G \alpha \Delta \varepsilon_{q} / q_{n}} \mathbf{J}+\left(K-\frac{2 G}{3} \frac{1}{1+3 G \alpha \Delta \varepsilon_{q} / q_{n}}\right) \mathbf{I} \otimes \mathbf{I}
$$

For the present pressure-dependent elastoplasticity model, using the same method as Aravas, ${ }^{16}$ by linearization of the flow (32a) and yield (32b) conditions, we obtain the following equations:

$$
\begin{aligned}
& C_{11} \partial \Delta \varepsilon_{p}+C_{12} \partial \Delta \varepsilon_{q}=\left(B_{11} \mathbf{I}+B_{12} \mathbf{n}_{n+\alpha}\right): \partial \boldsymbol{\sigma} \\
& C_{21} \partial \Delta \varepsilon_{p}+C_{22} \partial \Delta \varepsilon_{q}=\left(B_{21} \mathbf{I}+B_{22} \mathbf{n}_{n+1}\right): \partial \boldsymbol{\sigma}
\end{aligned}
$$


where the constants $C_{i j}$ and $B_{i j}$ are shown in Appendix II. Substitution of (42) into (44) gives

$$
\begin{aligned}
& \partial \Delta \varepsilon_{p}=\left(D_{11} \mathbf{I}+D_{12} \mathbf{n}_{n+\alpha}+D_{13} \mathbf{n}_{n+1}\right): \mathbf{Z}: \partial \boldsymbol{\varepsilon} \\
& \partial \Delta \varepsilon_{q}=\left(D_{21} \mathbf{I}+D_{22} \mathbf{n}_{n+\alpha}+D_{23} \mathbf{n}_{n+1}\right): \mathbf{Z}: \partial \boldsymbol{\varepsilon}
\end{aligned}
$$

where the constants $D_{i j}$ are given in Appendix III.

By substituting (45) into (42), we obtain an expression for the consistent tangent moduli with the generalized mid-point algorithms:

$$
\partial \boldsymbol{\sigma}=\mathbf{M}: \mathbf{Z}: \partial \boldsymbol{\varepsilon}
$$

where

$$
\begin{aligned}
\mathbf{M} & =\mathbf{J}-\mathbf{M}^{I}-\mathbf{M}^{n} \\
\mathbf{M}^{I} & =K\left(D_{11} \mathbf{I} \otimes \mathbf{I}+D_{12} \mathbf{I} \otimes \mathbf{n}_{n+\alpha}+D_{13} \mathbf{I} \otimes \mathbf{n}_{n+1}\right) \\
\mathbf{M}^{n} & =\frac{2 G}{\left(1+3 G \alpha \Delta \varepsilon_{q} / q_{n}\right)} \frac{q_{n+\alpha}}{q_{n}}\left(D_{21} \mathbf{n}_{n+\alpha} \otimes \mathbf{I}+D_{22} \mathbf{n}_{n+\alpha} \otimes \mathbf{n}_{n+\alpha}+D_{23} \mathbf{n}_{n+\alpha} \otimes \mathbf{n}_{n+1}\right)
\end{aligned}
$$

Finally, by multiplying $\mathbf{M}$ and $\mathbf{Z}$ and using the relationship between $\mathbf{I}$ and $\mathbf{n}$, we obtain the following explicit expression for the consistent tangent moduli:

$$
D^{\text {consis. }}=d_{0} \mathbf{J}+d_{1} \mathbf{I} \otimes \mathbf{I}+\mathbf{n}_{n+\alpha} \otimes\left(d_{2} \mathbf{n}_{n+\alpha}+d_{3} \mathbf{n}_{n+1}\right)+d_{4} \mathbf{n}_{n+\alpha} \otimes I+I \otimes\left(d_{5} \mathbf{n}_{n+\alpha}+d_{6} \mathbf{n}_{n+1}\right)
$$

where the seven constants are given by

$$
\begin{aligned}
& d_{0}=\frac{2 G}{1+3 G \alpha \Delta \varepsilon_{q} / q_{n}} \\
& d_{1}=K-\frac{d_{0}}{3}-3 K^{2} D_{11} \\
& d_{2}=-d_{0}^{2} \frac{q_{n+\alpha}}{q_{n}} D_{22} \\
& d_{3}=-d_{0}^{2} \frac{q_{n+\alpha}}{q_{n}} D_{23} \\
& d_{4}=-3 d_{0} K D_{21} \frac{q_{n+\alpha}}{q_{n}} \\
& d_{5}=-d_{0} K D_{12} \\
& d_{6}=-d_{0} K D_{13}
\end{aligned}
$$

It should be noted that no matrix inversion is involved in the present consistent tangent moduli (48). Equation (48) represents a general form of the consistent tangent moduli. It can be seen that there are two parts which make the consistent tangent moduli non-symmetric. The first part is attributed to the algorithm used and is represented by the third and last term of (48). Obviously, if and only if $\alpha=1$, then the third term makes no contribution to the non-symmetry of (48). Similar results for the ideal-plastic von Mises model found by Simo and Taylor ${ }^{4}$ and Ortiz and Martin ${ }^{23}$ show that only the fully implicit or the classical Euler backward algorithm preserves symmetry of the consistent tangent moduli. It is easy to see that if we enforce the plasticity consistency 
condition (16d) not at the end of the increment, but at mid-point time $t_{n+\alpha}$, i.e. $\phi_{n+\alpha}=0$, as suggested by Simo and Taylor ${ }^{4}$ and Simo and Govindjee ${ }^{24}$, then the third part of (48) is symmetric. However, it needs special formulations in real finite element application to enforce $\phi_{n+\alpha}=0$, because, by doing so, the momentum balance should also be enforced for the mid-point value stresses $\sigma_{n+\alpha}$. On the other hand, even for the algorithm where the consistency is enforced at time $t_{n+\alpha}$, i.e. $\phi_{n+\alpha}=0$, for example, the Euler backward algorithm $(\alpha=1)$, there is still one part represented by the last two terms of (48) which makes the consistent tangent moduli possibly non-symmetric $\left(d_{4} \neq d_{5}+d_{6}\right)$. The values of the last two terms of (48) depend on the material model. For the von Mises model with the classical Euler backward algorithm $(\alpha=1)$, it has been proved $^{17}$ that $d_{4}=d_{5}+d_{6}=0$. For the present pressure-dependent Gurson-Tvergaard material model, the non-symmetry of the consistent tangent moduli for the Euler backward algorithm has been demonstrated by numerical test. However, as pointed out by Aravas, ${ }^{16}$ the lack of symmetry is not expected to degrade the convergence of the Newton iterations for equilibrium if we approximate $\mathbf{D}^{\text {consis. }}$ with its symmetric part.

\section{NUMERICAL PERFORMANCE AND DISCUSSION}

The generalized mid-point algorithms described in the previous sections have been implemented with the explicit consistent tangent moduli in the general purpose finite element program ABAQUS $^{25}$ via the user material subroutine. The performance of the generalized mid-point algorithms has been assessed for two problems, plane strain tension and hydrostatic tension.

\subsection{Stress-strain curve of the matrix material}

The same uni-axial stress-strain relation as used by Aravas $^{16}$ is used for all the problems considered:

$$
\frac{\sigma_{f}}{\sigma_{f 0}}=\left(\frac{\sigma_{f}}{\sigma_{f 0}}+\frac{3 G}{\sigma_{f 0}} \bar{\varepsilon}^{\mathrm{p}}\right)^{\mathrm{N}}
$$

where $\sigma_{f 0}$ is the initial tensile yield stress of the matrix. The elastic-plastic properties of the matrix material are specified by $\sigma_{f o} / E=1 / 300, v=0.3, N=0.1, q_{1}=1.5$ and $q_{2}=1.0$. The void nucleation parameters in (5) are $f_{\mathrm{N}}=0.04, \varepsilon_{\mathrm{N}}=0.3$ and $S_{\mathrm{N}}=0 \cdot 1$.

\subsection{Plane strain tension problem}

A 8-node parabolic element with reduced integration (CPE8R) in ABAQUS was used for the finite element analysis. The element dimensions are shown in Figure 1, where $x, y$ are the symmetric axes. Displacement-controlled loading with equal displacement increment size was applied at the top of the element. The problem was solved with zero initial void volume fraction. The exact solution of the problem is numerically obtained by integrating the set of equations (9)-(12), using a Euler forward scheme with 200000 increments performed from the initial yield strain to a strain of 1.0 . The initial yield point in the loading path can be easily determined by using the analytical equations derived. There is no need to find the transition point between the elastic to the elastoplastic deformation in the present finite element implementation, because the implicit algorithms can find it automatically. In order to test the performance of the mid-point algorithms formulated in Section 5 , three values of $\alpha$ are chosen, $0.5,0.75$ and 1 . Two displacement increment sizes were tested in the finite element analysis. The comparisons of the stresses between the finite element results of both increment sizes and the numerically obtained exact 
results are shown in Table $I$ as a function of the logarithmic strain $\varepsilon_{y}=\ln \left(1+u / a_{0}\right)$, where $u$ is the prescribed $y$-direction displacement and $a_{0}$ is the initial element length. In Table $\mathrm{I}$, the results are compared at ten steps only, which cut the whole displacement history equally into ten. In the analysis using 80 increments, a negative system eigenvalue problem in the global Newton iteration appeared at increment 51 in the true mid-point algorithm $(\alpha=0.5)$ which discontinued the analysis. The asterisks in Table $I$ indicate that these values are not available because of the problem encountered.

It can be seen that even for the very large increment size (40 increments) applied, $\Delta \varepsilon_{y}$ (average) $=0.025=7 \cdot 5 \varepsilon_{f 0}$, the errors are not excessive for all the algorithms considered. It must be noted that, in the present solution, the increment is controlled by displacement. Therefore, it is difficult to obtain equal strain increments by displacement-controlled loading in large displacement non-linear analysis. Table I shows that for the plane strain tension problem the true mid-point algorithm $(\alpha=0.5)$ is the most accurate one in both small and large inrement sizes, except at the beginning of the analysis. It can be seen that the second-order accuracy of the true mid-point algorithm is not sensitive to the increment size in the present plane strain tension problem. By contrast, the Euler backward algorithm gives poorest accuracy, although the errors are not excessive at all. In general, the accuracy of the algorithms depends on the nature of the problem. In Reference 18, the accuracy of the generalized mid-point algorithms has been assessed by using different strain increment sizes and directions. In the assessment the initial stress location on the Gurson-Tvergaard yield surface $(f=0.03)$ was fixed at a point where the hydrostatic stress equals the initial yield stress. It was found that the second-order accuracy of the true mid-point has a limited range. In terms of the maximum error in all the increment sizes assessed, it was found that the optimal value of $\alpha$ is observed between 0.5 and 1 . When the deviatoric strain increment is radial to the yield surface, the true mid-point algorithm is usually the most accurate one, no matter how large the increment size. However, when the deviatoric strain increment is tangent to the yield surface, the second-order accuracy disappears once the strain increment size increases. For the present plane strain tension problem, the stress increases almost proportionally $(p / q \approx-0.55$, point $A$ in Figure 3$)$ and the deviatoric strain increment is radial to the yield surface. Therefore, the strain increment scheme in the plane strain tension problem lies in the

Table I. Comparison of the stress $\sigma_{y}$ between the FE results and the exact results for plane strain tension problem. 40 and 80 equal displacement increments were applied in FE solution

\begin{tabular}{|c|c|c|c|c|c|c|c|c|}
\hline & \multicolumn{2}{|c|}{ Exact values } & \multicolumn{3}{|c|}{ FEM -40 increments } & \multicolumn{3}{|c|}{ FEM -80 increments } \\
\hline$\varepsilon_{y}$ & $\begin{array}{c}\text { Exact } \\
\text { stress } \\
\sigma_{y}\end{array}$ & $\begin{array}{c}\text { Exact } \\
f\end{array}$ & $\begin{array}{c}\alpha=0.5 \\
\text { error } \\
(\%)\end{array}$ & $\begin{array}{c}\alpha=0.75 \\
\text { error } \\
(\%)\end{array}$ & $\begin{array}{l}\alpha=1 \\
\text { error } \\
(\%)\end{array}$ & $\begin{array}{c}\alpha=0.5 \\
\text { error } \\
(\%)\end{array}$ & $\begin{array}{c}\alpha=0.75 \\
\text { error } \\
(\%)\end{array}$ & $\begin{array}{c}\alpha=1 \\
\text { error } \\
(\%)\end{array}$ \\
\hline 0.159 & 172.9 & 0.0048 & -0.87 & -0.58 & -0.52 & -0.35 & -0.29 & -0.29 \\
\hline 0.295 & $174 \cdot 2$ & 0.0306 & 0.23 & -0.52 & $-1 \cdot 32$ & $0 \cdot 17$ & -0.23 & -0.69 \\
\hline 0.416 & $170 \cdot 8$ & 0.0553 & 0.23 & -0.59 & $-1 \cdot 46$ & 0.12 & -0.29 & -0.70 \\
\hline 0.523 & 168.6 & 0.0714 & 0.24 & -0.71 & -1.72 & 0.12 & -0.36 & -0.83 \\
\hline 0.620 & $165 \cdot 7$ & 0.0868 & 0.36 & -0.84 & $-2 \cdot 11$ & $0 \cdot 18$ & -0.42 & -1.03 \\
\hline 0.709 & $161 \cdot 9$ & 0.1027 & 0.49 & -0.93 & $-2 \cdot 47$ & 0.25 & -0.43 & -1.17 \\
\hline 0.790 & 157.6 & $0 \cdot 1191$ & 0.63 & -1.02 & $-2 \cdot 79$ & * & -0.51 & -1.40 \\
\hline 0.865 & 152.9 & $0 \cdot 1358$ & 0.78 & $-1 \cdot 11$ & $-3 \cdot 14$ & * & -0.59 & -1.50 \\
\hline 0.935 & 148.0 & $0 \cdot 1526$ & 0.81 & -1.28 & -3.51 & * & -0.68 & -1.76 \\
\hline 1.000 & $143 \cdot 1$ & 0.1693 & 0.98 & -1.33 & $-3 \cdot 77$ & * & -0.70 & -1.89 \\
\hline
\end{tabular}




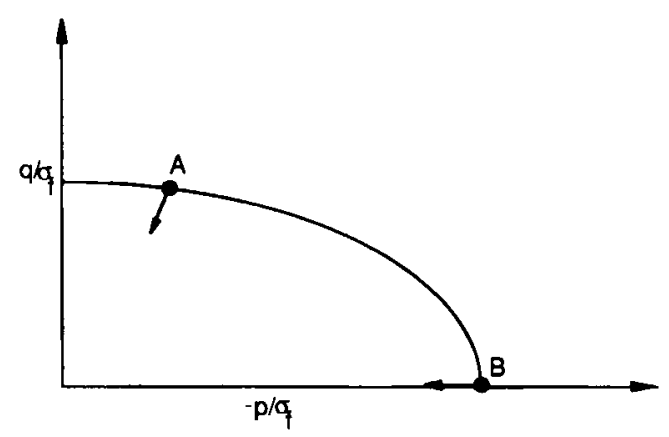

Figure 3. Locations on the yield surface: (A) plane strain tension problem; (B) hydrostatic tension problem

second-order accuracy range of the true mid-point algorithm observed in Reference 18 . The second-order accuracy of the true mid-point algorithm demonstrated by the plane strain tension problem has further proved the observation in Reference 18. These findings could lead to some general conclusions which will be stated later.

Figures 4 and 5 show the comparisons between the stress and void volume fraction results of the Euler backward algorithm $(\alpha=1)$ and the exact results. Although the Euler backward algorithm is not the most accurate one in the plane strain tension problem, very good accuracy has been demonstrated within the global behaviour, especially when the increment size is small.

In ABAQUS, we only used the symmetric part of the consistent tangent moduli for global iteration, even though the convergence behaviour of all the algorithms assessed is very good. Table II shows the number of global iterations used in the large increment size ( 40 increments) analysis. It is seen that on average less than two iterations for each increment were needed and the true mid-point algorithm is the fastest one to converge and is much faster than the Euler backward algorithm. In the 80 increment analysis, there is no big difference in the convergence behaviour of the three algorithms, because the increment size is relatively small.

\subsection{Hydrostatic tension problem}

An 8-node brick element with reduced integration (C3D8R) in ABAQUS was used for testing the hydrostatic tension problem. The dimensions of the problem are shown in Figure 2. The boundary conditions are the same as those in Reference 16. Equal displacement increments are used so that a logarithmic volumetric strain of 0.4 is reached in 10 and 20 increments, respectively. The initial void volume fraction is 0.04 , all the other parameters are the same as the plane strain tension problem. The finite element analysis results of three algorithms are compared with the numerically obtained exact results for two different increment sizes in Table III. Similar to the plane strain tension problem, the results of the three algorithms agree very well with the exact results. It is interesting to note that for the hydrostatic tension problem, in which only the volumetric strain increment is available and the strain increment is exactly perpendicular to the yield surface ( $q=0$, point B in Figure 3), the accuracy of the generalized algorithms varies with the change of void volume fraction or the curvature of the yield surface at point B in Figure 3. In the beginning when the void volume fraction is small, the Euler backward algorithm is the most accurate one; however when the void volume fraction exceeds about 0.2 , the true mid-point algorithm becomes the most accurate one. For both large and small increment sizes, the algorithm with $\alpha=0.75$ gives moderate accuracy during the whole history of the analysis. 


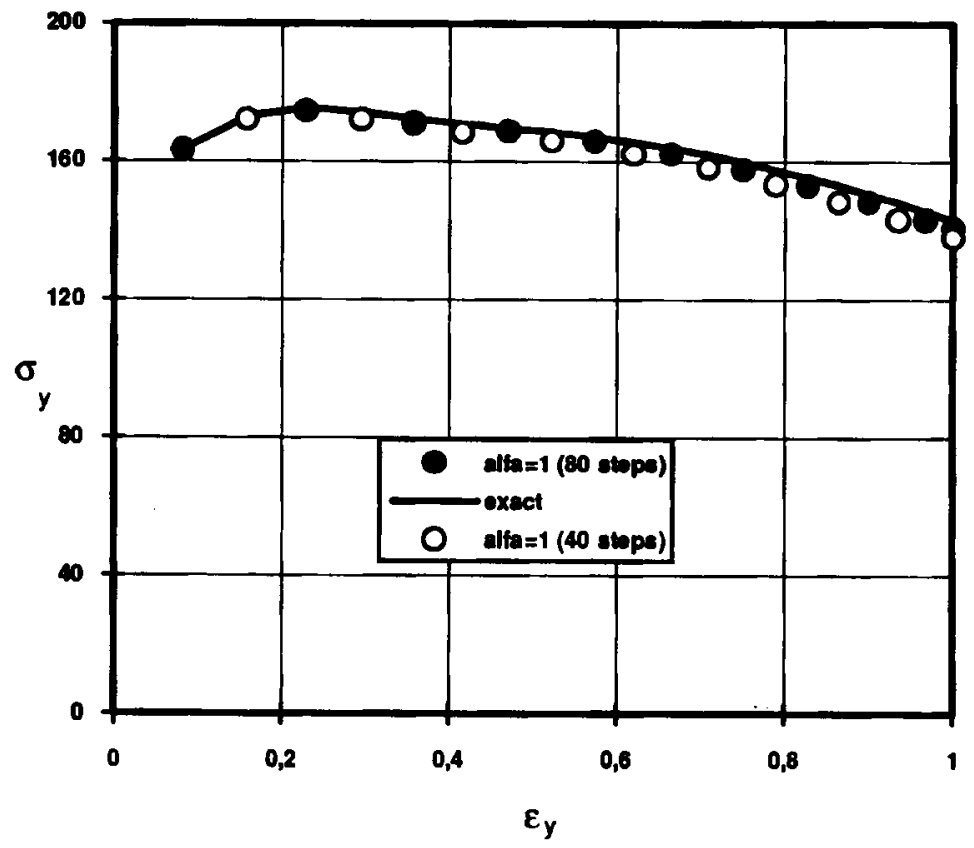

Figure 4. Plane strain tension stress-strain curves

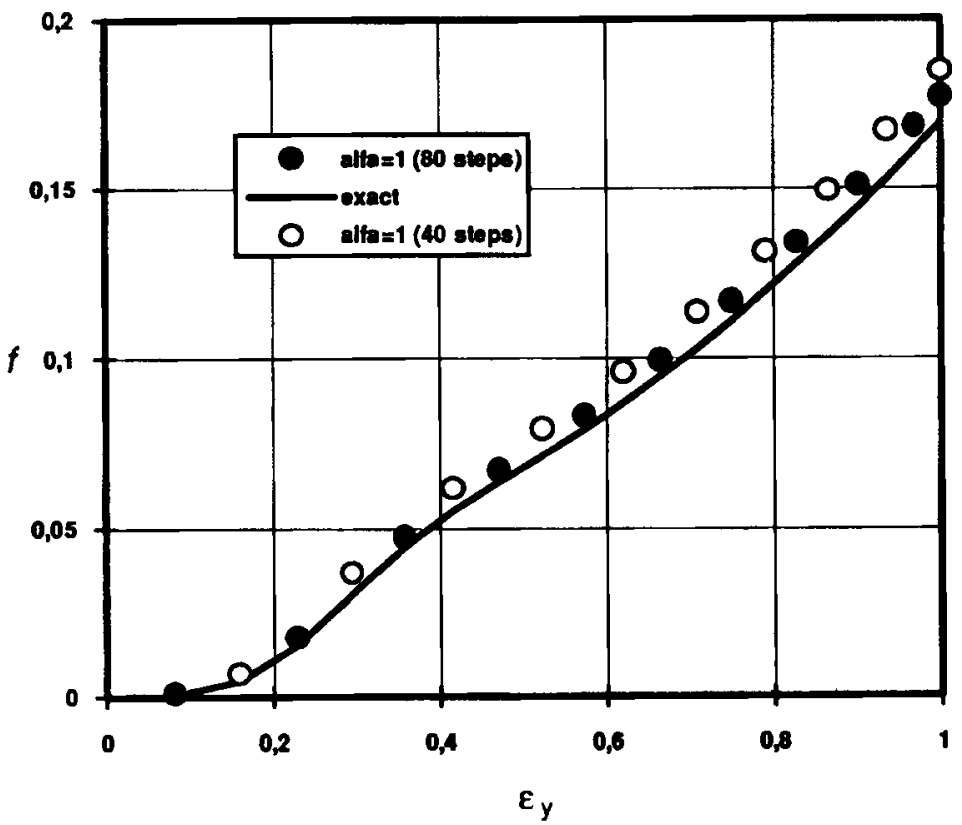

Figure 5. Plane strain tension: porosity as a function strain 
The finite element analysis results of the hydrostatic stress and void volume fraction for the Euler backward algorithm $(\alpha=1)$ using large increment steps are plotted as a function of $\varepsilon_{\mathrm{v}}$ in Figures 6 and 7, respectively. It can be seen that finite element results agree very well with the exact results, even when using very large increment steps. Due to the absence of deviatoric stress, the convergence in the global Newton iteration is very fast, only one iteration is needed in every increment, regardless of the algorithm chosen.

\subsection{General remarks}

From the numerical behaviour of the present finite element results and the observation in Reference 18 , it can be concluded that the accuracy of the generalized mid-point algorithms depends on many factors, for example, the location of stress points on the yield surface, the yield surface curvature, the strain increment direction to the yield surface and the strain increment size. The second-order accuracy of the true mid-point algorithm depends strongly on the direction of the strain increment to the yield surface. As shown in the analysed plane strain tension case, the second-order accuracy of the true mid-point algorithm is almost insensitive to the strain increment size if the deviatoric strain increment is radial to the yield surface. Similar findings can be seen in the results of the von Mises model by Ortiz and Popov. ${ }^{2}$ Therefore, it can be generally concluded that for the loading case, where the deviatoric strain increment is radial to the yield surface, the true mid-point algorithm is the most accurate one. The results in the plane strain tension problem have also shown that the true mid-point algorithm is not only the most accurate, but also the fastest in convergence in global Newton iteration. However, one thing which is

Table II. Number of iterations in the $\mathbf{4 0}$ increment analysis

\begin{tabular}{lccc}
\hline Algorithms & $\alpha=0.5$ & $\alpha=0.75$ & $\alpha=1$ \\
\hline No. of iterations & 43 & 61 & 65 \\
\hline
\end{tabular}

Table III. Comparison of hydrostatic pressure between FE results and the exact results for hydrostatic tension problem. 10 and 20 equal displacement increments were applied in FE solution

\begin{tabular}{|c|c|c|c|c|c|c|c|c|}
\hline & \multicolumn{2}{|c|}{ Exact values } & \multicolumn{3}{|c|}{ FEM -10 increments } & \multicolumn{3}{|c|}{ FEM-20 increments } \\
\hline$\varepsilon_{p}$ & $\begin{array}{l}\text { Exact } \\
(-p)\end{array}$ & $\begin{array}{c}\text { Exact } \\
f\end{array}$ & $\begin{array}{c}\alpha=0.5 \\
\text { error } \\
(\%)\end{array}$ & $\begin{array}{c}\alpha=0.75 \\
\text { error } \\
(\%)\end{array}$ & $\begin{array}{l}\alpha=1 \\
\text { error } \\
(\%)\end{array}$ & $\begin{array}{c}\alpha=0.5 \\
\text { error } \\
(\%)\end{array}$ & $\begin{array}{c}\alpha=0.75 \\
\text { error } \\
(\%)\end{array}$ & $\begin{array}{l}\alpha=1 \\
\text { error } \\
(\%)\end{array}$ \\
\hline 0.042 & $201 \cdot 4$ & 0.0728 & $-6 \cdot 70$ & $-3 \cdot 23$ & -0.65 & $-2 \cdot 23$ & $-1 \cdot 19$ & -0.35 \\
\hline 0.084 & $172 \cdot 8$ & $0 \cdot 1130$ & $-2 \cdot 60$ & -1.39 & -0.46 & -0.93 & -0.58 & -0.29 \\
\hline $0 \cdot 126$ & $148 \cdot 7$ & 0.1524 & $-1 \cdot 14$ & -0.54 & -0.2 & -0.34 & $-0 \cdot 20$ & -0.13 \\
\hline 0.166 & 128.8 & 0.1911 & -0.23 & 0.00 & 0.08 & -0.08 & 0.00 & 0.00 \\
\hline 0.207 & $112 \cdot 1$ & 0.2287 & 0.36 & 0.45 & 0.45 & $0 \cdot 18$ & $0 \cdot 18$ & 0.18 \\
\hline 0.246 & 98.1 & 0.2645 & 0.67 & 0.72 & 0.76 & 0.25 & 0.31 & 0.37 \\
\hline 0.285 & $86 \cdot 3$ & 0.2980 & 0.75 & 0.92 & $1 \cdot 10$ & 0.27 & 0.39 & 0.54 \\
\hline 0.324 & $76 \cdot 3$ & 0.3290 & 0.69 & 1.04 & 1.40 & 0.25 & 0.47 & 0.72 \\
\hline 0.362 & 67.8 & 0.3575 & 0.46 & 1.02 & 1.61 & $0 \cdot 13$ & 0.47 & $0-80$ \\
\hline 0.400 & $60 \cdot 4$ & 0.3838 & 0.26 & 1.04 & 1.85 & 0.08 & 0.51 & 0.94 \\
\hline
\end{tabular}




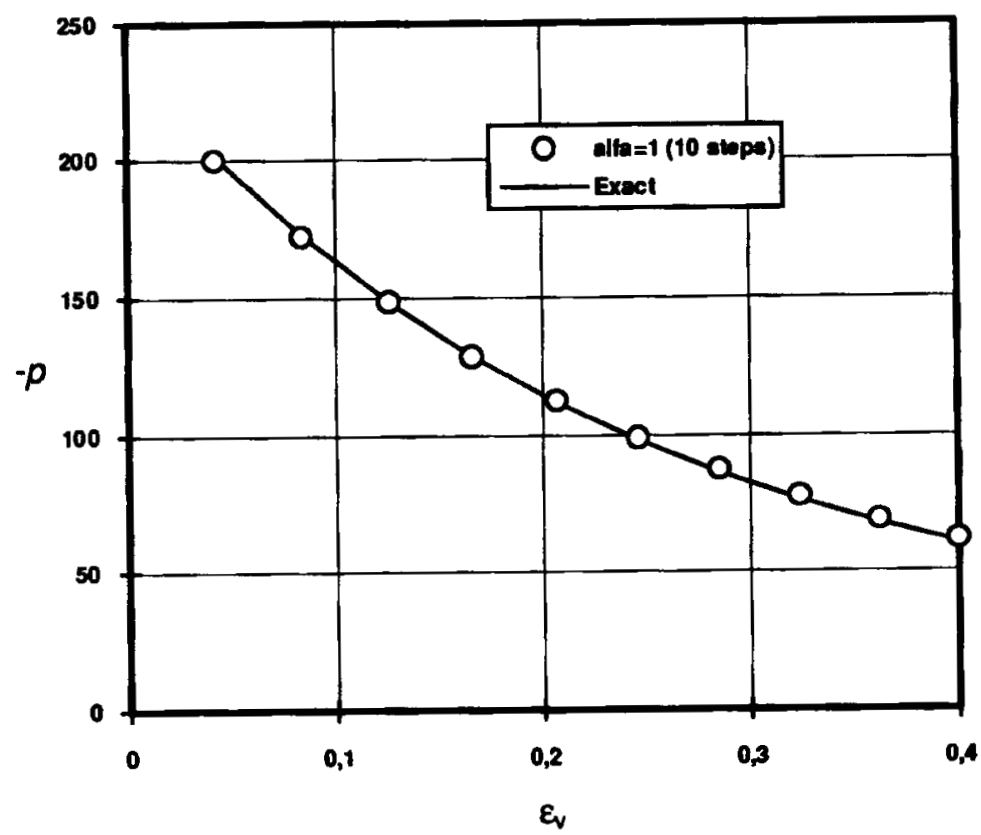

Figure 6. Pressure as a function of $\varepsilon_{\mathrm{v}}$

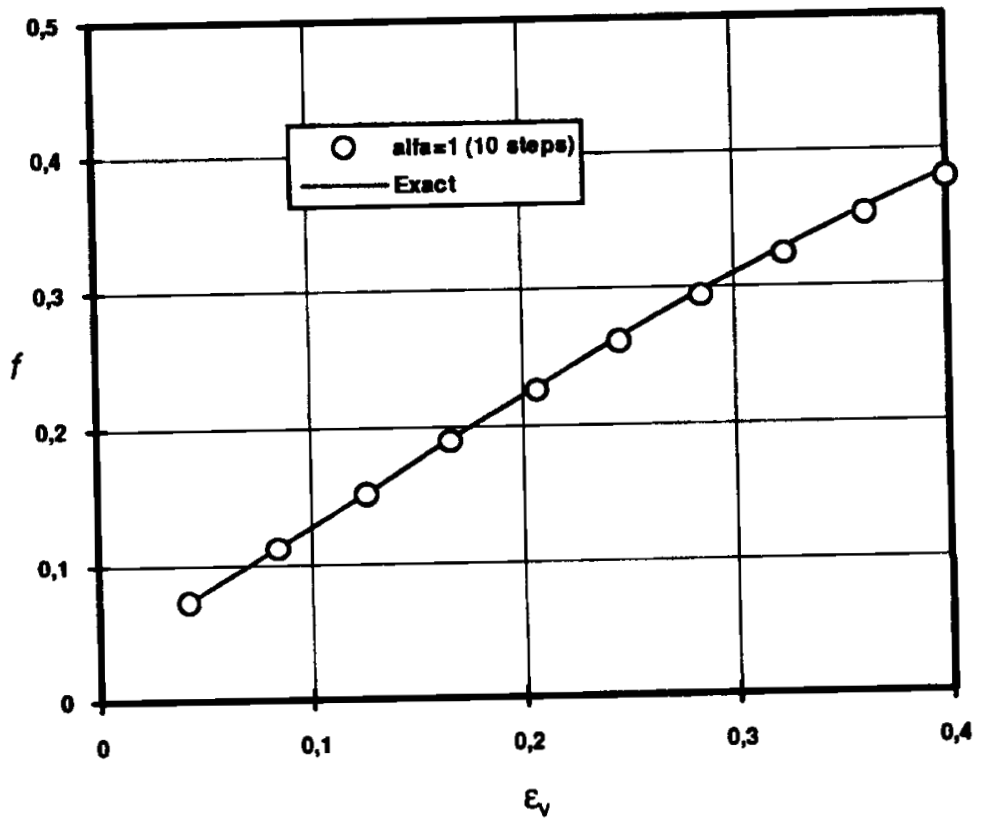

Figure 7. Void volume fraction as a function of $\varepsilon_{v}$ 
necessary to pay attention to is that, although the true mid-point algorithm is the most accurate and efficient one in the plane strain tension problem simulated by one element, an unexpected negative system eigenvalue problem once appeared in the true mid-point algorithm which discontinued the analysis (see Table I). The reason why the negative eigenvalue problem appeared only in the true mid-point algorithm was not clearly known to us; however, it may have something to do with the extra non-symmetry caused by the algorithm, as discussed in Section 5. We have not tried to enforce the consistent condition at time $t_{n+a}$, i.e. $\phi_{n+a}=0$, yet, to avoid the extra non-symmetry. Although we have not experienced the negative system eigenvalue problem in any other applications of the true mid-point algorithm, where the stress distribution was not uniform and more than one element was used, considering this incident of the true mid-point algorithm and the fact that the Euler backward algorithm has no extra non-symmetric term caused by the algorithm and is versatile, reasonably accurate, and has been widely used in the von Mises model, the Euler backward algorithm should be recommended.

\section{CONCLUSIONS}

A class of generalized mid-point algorithms for the increasingly popular Gurson-Tvergaard model has been presented and incorporated into the finite element program ABAQUS via user material subroutine. By decomposing the stress into hydrostatic and deviatoric parts, a sevenconstant formula for calculating the corresponding consistent tangent moduli with the generalized mid-point algorithms has been presented. The formula presented in the paper is simple and can be regarded as a general form of the consistent tangent moduli. One interesting feature of the formula is that the non-symmetric property of the consistent tangent moduli can be easily identified and studied. The performance and accuracy of the generalized mid-point algorithms have been assessed by application to two cases, plane strain and hydrostatic tension problems. Results show that in all the cases considered, the class of the generalized mid-point algorithms is reasonably accurate. Furthermore, the accuracy of the algorithms seems to depend on many factors and it has been concluded that for the case where the deviatoric strain increment is radial to the yield surface, the true mid-point algorithm is the most accurate. However, considering the extra non-symmetric property of the consistent tangent moduli caused by the algorithms with $\alpha<1.0$, and the negative eigenvalue problem encountered in this study for the true mid-point algorithm, the Euler backward algorithm $(\alpha=1)$ is perhaps the best choice for the wide application of the Gurson-Tvergaard model.

\section{ACKNOWLEDGEMENT}

The support from the Ministry of Education of Finland is gratefully acknowledged.

\section{APPENDIX I}

\section{Coefficients in equation (33)}

Treating $\Delta \varepsilon_{p}$ and $\Delta \varepsilon_{q}$ as the two independent unknowns and all other variables as a function of $\Delta \varepsilon_{p}$ and $\Delta \varepsilon_{q}$, and using Newton's method to solve (32a) and (32b), we obtain the following coefficients in (33):

$$
A_{11}=P+\alpha \Delta \varepsilon_{p}\left[\frac{\partial P}{\partial p_{n+\alpha}} \frac{\partial p_{n+1}}{\partial \Delta \varepsilon_{p}}+\frac{\partial P}{\partial \mathbf{H}_{n+\alpha}}: \frac{\partial \mathbf{H}_{n+1}}{\partial \Delta \varepsilon_{p}}\right]+\alpha \Delta \varepsilon_{q}\left[\frac{\partial Q}{\partial p_{n+\alpha}} \frac{\partial p_{n+1}}{\partial \Delta \varepsilon_{p}}+\frac{\partial Q}{\partial \mathbf{H}_{n+\alpha}}: \frac{\partial \mathbf{H}_{n+1}}{\partial \Delta \varepsilon_{p}}\right]
$$




$$
\begin{aligned}
A_{12}= & Q+\alpha \Delta \varepsilon_{p}\left[\frac{\partial P}{\partial q_{n+\alpha}} \frac{3}{2} \frac{\mathbf{S}_{n+\alpha}}{q_{n+\alpha}}: \frac{\partial \mathbf{S}_{n+1}}{\partial \Delta \varepsilon_{q}}+\frac{\partial P}{\partial \mathbf{H}_{n+\alpha}}: \frac{\partial \mathbf{H}_{n+1}}{\partial \Delta \varepsilon_{p}}\right] \\
& +\alpha \Delta \varepsilon_{q}\left[\frac{\partial Q}{\partial q_{n+\alpha}} \frac{3}{2} \frac{\mathbf{S}_{n+\alpha}}{q_{n+\alpha}}: \frac{\partial \mathbf{S}_{n+1}}{\partial \Delta \varepsilon_{q}}+\frac{\partial Q}{\partial \mathbf{H}_{n+\alpha}}: \frac{\partial \mathbf{H}_{n+1}}{\partial \Delta \varepsilon_{q}}\right] \\
A_{21}= & \frac{\partial \phi_{n+1}}{\partial p_{n+1}} \frac{\partial p_{n+1}}{\partial \Delta \varepsilon_{p}}+\frac{\partial \phi_{n+1}}{\partial \mathbf{H}_{n+1}}: \frac{\partial \mathbf{H}_{n+1}}{\partial \Delta \varepsilon_{p}} \\
A_{22}= & \frac{\partial \phi_{n+1}}{\partial q_{n+1}} \frac{\partial q_{n+1}}{\partial \Delta \varepsilon_{q}}+\frac{\partial \phi_{n+1}}{\partial \mathbf{H}_{n+1}}: \frac{\partial \mathbf{H}_{n+1}}{\partial \Delta \varepsilon_{q}} \\
b_{1}= & -\Delta \varepsilon_{p} P-\Delta \varepsilon_{q} Q \\
b_{2}= & -\phi_{n+1}
\end{aligned}
$$

where we have

$$
\begin{aligned}
& \frac{\partial H_{n+1}^{i}}{\partial \Delta \varepsilon_{p}}=w_{i j}\left(\frac{\partial h_{n+\alpha}^{j}}{\partial \Delta \varepsilon_{p}}+\frac{\partial h_{n+\alpha}^{j}}{\partial p_{n+\alpha}} \frac{\partial p_{n+\alpha}}{\partial \Delta \varepsilon_{p}}\right) \\
& \frac{\partial H_{n+1}^{i}}{\partial \Delta \varepsilon_{q}}=w_{i j}\left(\frac{\partial h_{n+\alpha}^{j}}{\partial \Delta \varepsilon_{q}}+\frac{\partial h_{n+\alpha}^{j}}{\partial q_{n+\alpha}} \frac{\partial q_{n+\alpha}}{\partial \Delta \varepsilon_{q}}\right)
\end{aligned}
$$

Here $w_{i j}$ is the inverse of

$$
\delta_{i j}-\alpha \frac{\partial h_{n+\alpha}^{i}}{\partial H_{n+\alpha}^{j}}
$$

and for the Gurson-Tvergaard model applied in the paper, $2 \geqslant i, j \geqslant 1$.

\section{APPENDIX II}

\section{Coefficients in equation (44)}

By linearization of (32a) and (32b) with respect to $\partial \Delta \varepsilon_{p}, \partial \Delta \varepsilon_{q}$ and $\partial \sigma(p, q)$, the coefficients in (44) which relate $\partial \Delta \varepsilon_{p}, \partial \Delta \varepsilon_{q}$ and $\partial \sigma$ are obtained:

$$
\begin{aligned}
& C_{11}=P+\left[\Delta \varepsilon_{p} \frac{\partial P}{\partial \mathbf{H}_{n+\alpha}}+\Delta \varepsilon_{q} \frac{\partial Q}{\partial \mathbf{H}_{n+\alpha}}\right] \alpha \frac{\partial \mathbf{H}_{n+1}}{\partial \Delta \varepsilon_{p}} \\
& C_{12}=Q+\left[\Delta \varepsilon_{p} \frac{\partial P}{\partial \mathbf{H}_{n+\alpha}}+\Delta \varepsilon_{q} \frac{\partial Q}{\partial \mathbf{H}_{n+\alpha}}\right] \alpha \frac{\partial \mathbf{H}_{n+1}}{\partial \Delta \varepsilon_{q}} \\
& B_{11}=\frac{\alpha}{3} \Delta \varepsilon_{p}\left[\frac{\partial P}{\partial p_{n+\alpha}}+\frac{\partial P}{\partial \mathbf{H}_{n+\alpha}} \frac{\partial \mathbf{H}_{n+1}}{\partial p_{n+\alpha}} \alpha\right]+\frac{\alpha}{3} \Delta \varepsilon_{q}\left[\frac{\partial Q}{\partial p_{n+\alpha}}+\frac{\partial Q}{\partial \mathbf{H}_{n+\alpha}} \frac{\partial \mathbf{H}_{n+1}}{\partial p_{n+\alpha}} \alpha\right] \\
& B_{12}=-\alpha \Delta \varepsilon_{p}\left[\frac{\partial P}{\partial q_{n+\alpha}}+\frac{\partial P}{\partial \mathbf{H}_{n+\alpha}} \frac{\partial \mathbf{H}_{n+1}}{\partial q_{n+\alpha}} \alpha\right]-\alpha \Delta \varepsilon_{q}\left[\frac{\partial Q}{\partial q_{n+\alpha}}+\frac{\partial Q}{\partial \mathbf{H}_{n+\alpha}} \frac{\partial \mathbf{H}_{n+1}}{\partial q_{n+\alpha}} \alpha\right] \\
& C_{21}=\frac{\partial \phi_{n+1}}{\partial \mathbf{H}_{n+1}}: \frac{\partial \mathbf{H}_{n+1}}{\partial \Delta \varepsilon_{p}} \\
& C_{22}=\frac{\partial \phi_{n+1}}{\partial \mathbf{H}_{n+1}}: \frac{\partial \mathbf{H}_{n+1}}{\partial \Delta \varepsilon_{q}}
\end{aligned}
$$




$$
\begin{aligned}
& B_{21}=\frac{1}{3}\left[\frac{\partial \phi_{n+1}}{\partial p_{n+1}}+\frac{\partial \phi_{n+1}}{\partial \mathbf{H}_{n+1}}: \frac{\partial \mathbf{H}_{n+1}}{\partial p_{n+1}}\right] \\
& B_{22}=-\left[\frac{\partial \phi_{n+1}}{\partial q_{n+1}}+\frac{\partial \phi_{n+1}}{\partial \mathbf{H}_{n+1}}: \frac{\partial \mathbf{H}_{n+1}}{\partial q_{n+1}}\right]
\end{aligned}
$$

where we have

$$
\begin{aligned}
& \frac{\partial H_{n+1}^{i}}{\partial \Delta \varepsilon_{p}}=w_{i j} \frac{\partial h_{n+\alpha}^{j}}{\partial \Delta \varepsilon_{p}} \\
& \frac{\partial H_{n+1}^{i}}{\partial \Delta \varepsilon_{q}}=w_{i j} \frac{\partial h_{n+\alpha}^{j}}{\partial \Delta \varepsilon_{q}} \\
& \frac{\partial H_{n+1}^{i}}{\partial p_{n+\alpha}}=w_{i j} \frac{\partial h_{n+\alpha}^{j}}{\partial p_{n+\alpha}} \\
& \frac{\partial H_{n+1}^{i}}{\partial q_{n+\alpha}}=w_{i j} \frac{\partial h_{n+\alpha}^{j}}{\partial q_{n+\alpha}}
\end{aligned}
$$

and for the Gurson-Tvergaard model applied in the paper, $2 \geqslant i, j \geqslant 1$. In the derivation, $\partial q_{n+\alpha} / \partial \boldsymbol{\sigma}_{n+1}=\alpha \mathbf{n}_{n+\alpha}$ has been used.

\section{APPENDIX III}

Coefficients in equation (45)

$$
\begin{aligned}
& D_{11}=\left[\left(C_{22}+3 \bar{G} * B_{22}\right) * B_{11}-\left(C_{12}+3 \bar{G} * B_{12}\right) * B_{21}\right] / \Omega \\
& D_{12}=\left[\left(C_{22}+3 \bar{G} * B_{22}\right) * B_{12}\right] / \Omega \\
& D_{13}=\left[-\left(C_{12}+3 \bar{G} * B_{12}\right) * B_{22}\right] / \Omega \\
& D_{21}=\left[\left(C_{11}+3 K * B_{11}\right) * B_{21}-\left(C_{21}+3 K * B_{21}\right) * B_{11}\right] / \Omega \\
& D_{22}=\left[-\left(C_{21}+3 K * B_{21}\right) * B_{12}\right] / \Omega \\
& D_{23}=\left[\left(C_{11}+3 K * B_{11}\right) * B_{22}\right] / \Omega
\end{aligned}
$$

where

$$
\begin{aligned}
& \Omega=\left(C_{11}+3 K B_{11}\right) *\left(C_{22}+3 \bar{G} B_{22}\right)-\left(C_{21}+3 K B_{21}\right) *\left(C_{12}+3 \bar{G} B_{12}\right) \\
& \bar{G}=\frac{2 G / 3}{\left(1+3 G \alpha \Delta \varepsilon_{q} / q_{n}\right)} \frac{q_{n+\alpha}}{q_{n}} \mathbf{n}_{n+\alpha}: \mathbf{n}_{n+1}
\end{aligned}
$$

$G$ is the shear modulus.

\section{REFERENCES}

1. M. Ortiz and J. C. Simo, 'An analysis of a new class of integration algorithm for elastoplastic constitutive relations', Int. $j$. numer. methods eng., 23, 353-366 (1986).

2. M. Ortiz and E. P. Popov, 'Accuracy and stability of integration algorithms for elastoplastic constitutive relations', Int. j. numer. methods eng., 21, 1561-1576 (1985).

3. P. Gratacos, P. Montmitonnet and J. L. Chenot, 'An integration scheme for Prandtl-Reuss elastoplastic constitutive equations', Int. j. numer. methods eng., 33, 943-961 (1992). 
4. J. C. Simo and R. L. Taylor, 'A return mapping algorithm for plane stress elastoplasticity', Int. j. numer. methods eng., 22, 649-670 (1986).

5. J. C. Simo and R. L. Taylor, 'Consistent tangent operators for rate-independent elastoplasticity', Comput. Methods Appl. Mech. Eng., 48, 101-118 (1985).

6. J. C. Simo and M. Ortiz, 'A unified approach to finite deformation elastoplastic analysis based on the use of hyperelastic constitutive equations', Comput. Methods Appl. Mech. Eng., 49, 221-245 (1985).

7. A. L. Gurson, 'Plastic flow and fracture behaviour of ductile materials incorporating void nucleation, growth, and interaction', Ph.D. Dissertation, Brown University, 1975.

8. A. L. Gurson, 'Continuum theory of ductile rupture by void nucleation and growth: part I - yield criteria and flow rules for porous ductile media', J. Eng. Mater. Tech., 99, 2-15 (1977).

9. V. Tvergaard, 'Influence of voids on shear band instabilities under plane strain conditions', Int. J. Fract., 17, 389-407 (1981).

10. V. Tvergaard, 'On localization in ductile materials containing spherical voids', Int. J. Fract., 18, 237--252 (1982).

11. M. E. Mear, 'On the plastic yielding of porous metals', Mech. Mater., 9, 33-48 (1990).

12. M. Saja, J. Pan and A. Needleman, 'Void nucleation effects on shear localization in porous plastic solids', Int. J. Fract., 19, 163-182 (1982).

13. V. Tvergaard and A. Needleman, 'Analysis of the cup-cone fracture in a round tensile bar', Acta Metall., 32, 157-169 (1984).

14. M. W. Heinstein and H. T. Y. Yang, 'Plane strain finite element simulation of shear band formation during meta forming', Int. j. numer. methods eng., 33, 719-737 (1992).

15. M. J. Worswick and R. J. Pick, 'Void growth in plastically-deformed free-cutting brass', J. Appl. Mech. ASME, 58, $631-638$ (1991).

16. N. Aravas, 'On the numerical integration of a class of pressure-dependent plasticity models', Int. j. numer. methods eng., 24, 1395-1416 (1987).

17. Z. L. Zang, 'A practical micro-mechanical model-based local approach methodology for the analysis of ductile fracture of welded joints', Ph.D. Thesis, Lappeenranta University of Technology, 1994.

18. Z. L. Zhang, 'On the accuracies of numerial integration algorithms for Gurson pressure-dependent elastoplastic constitutive models', Comput. Methods Appl. Mech. Eng., to appear.

19. A. Needleman and V. Tvergaard, 'Analysis of plastic flow localization in metals', Appl. Mech. Rev., 45, s3-s18 (1992).

20. Y. Sun, 'The microscopic constitutive theory of ductility damaged metals and its application to instability and fracture analysis', Ph.D. Thesis, Harbin Institute of Technology, China, 1989.

21. X. Q. Yan, 'Effect of yield surface curvature on local necking in biaxially stretched sheets in porous materials', J. Eng., Mater. Tech., 114, 196-200 (1992).

22. C. C. Chu and A. Needleman, 'Void nucleation effects in biaxially stretched sheets', J. Eng. Mater. Tech., 102, 249-256 (1980).

23. M. Ortiz and J. B. Martin, 'Symmetry-preserving return mapping algorithms and incrementally extremal paths: a unification of concepts', Int. j. numer. methods eng., 28, 1839-1853 (1989).

24. J. C. Simo and S. Govindijee, 'Non-linear B-stability and symmetry preserving return mapping algorithms for plasticity and viscoplasticity', Int. j. numer. methods eng., 31, 151-176 (1991).

25. ABAQUS User's Manual, V5.2, Hibbit, Karlsson \& Sorensen, Inc., 1992. 